\title{
Public utility or places of experiment? The reality of the asylum
}

\section{Pubblica utilità o luoghi d'esperimento? La realtà manicomiale ¿Servicio público o lugares de experimentación? La realidad del manicomio}

\author{
Carmine Caputo \\ Dottore in Sociologia e Criminologia e in Investigazione, Criminalità e Sicurezza Internazionale, Università degli Studi \\ Internazionali di Roma, Italy
}

\begin{abstract}
"
The intention of this paper is to provide the most thorough overview of what concerns the reality of the asylum, through different types of analysis, in order to outline a detailed historical, political, health and cultural picture of the phenomenon. The historical and sociopsychological analysis in the paper traces the stages of the stigma of the "insane" (since antiquity, in fact, insanity and diversity have been two of the most unknown and frequent problems in the history of mankind) from the Middle Ages to the present day, a very long period of time in which there have been more and more interpretations on the subject; suffice it to say that now we speak of "mental illness", while years ago the mentally ill person was referred to by most as "crazy", a victim of insanity, an incurable disease. Following the analysis is carried out from a social and political point of view with a focus on the darkest and hidden aspects of asylums "Goffmanian" defined as "total institutions". In this sense, the work will focus on the various controversies and protests that have taken place over the years, and on the consequent laws taken on the subject, up to the Basaglia Law of 1978. It will also examine the internal organization of asylums, their structure bare and free of disturbing elements for patients, the training practices of staff, without of course neglecting the condition, the day type of patients and especially the "special treatments" reserved for patients. The flagship of this work is an interview with a former nurse from the largest asylum in Italy, the Mombello asylum, which aims to collect a testimony as it can be direct, engaging and above all not devoid of empirical references.
\end{abstract}

\section{RIASSUNTO}

L'obiettivo del presente lavoro è quello di fornire una panoramica quanto più completa su ciò che concerne la realtà manicomiale, attraverso diversi tipi di analisi, al fine di delineare un dettagliato quadro storico, politico, sanitario e culturale del fenomeno. L'analisi storico-sociopsicologica presente nell'elaborato ripercorre le tappe dello stigma del "folle" (fin dall'antichità infatti follia e diversità sono stati due tra i problemi più ignoti e frequenti nella storia dell'umanità) dal Medioevo sino ai giorni nostri, un ampissimo lasso di tempo in cui ci sono state sempre più interpretazioni in materia; basti pensare che ora si parla di "malattia mentale", mentre anni addietro il malato mentale era additato dai più come "folle", vittima della follia, malattia incurabile. A seguire l'analisi è svolta da un punto di vista sociale e politico con focus sugli aspetti più bui e celati dei manicomi "Goffmanianamente" definiti "istituzioni totali". In tal senso il lavoro sarà incentrato sulle varie controversie e proteste susseguitesi negli anni, e sulle conseguenti leggi prese in materia, sino ad arrivare alla Legge Basaglia del 1978. Verrà inoltre approfondita la tematica sull'organizzazione interna dei manicomi, sulla loro struttura scarna e priva di elementi di disturbo per i degenti, sulle pratiche di formazione del personale, senza ovviamente tralasciare condizione, giornata tipo degli internati e soprattutto i "trattamenti speciali" riservati agli internati. Fiore all'occhiello del presente lavoro è un'intervista ad un'ex infermiera del più grande manicomio d'Italia, il manicomio di Mombello (MB), che ha come scopo quello di raccogliere una testimonianza quanto più diretta, coinvolgente e soprattutto non priva di riferimenti empirici.

\section{RESUMEN}

El objetivo de este trabajo es ofrecer una visión lo más completa posible de lo que concierne a la realidad de los manicomios, a través de diferentes tipos de análisis, con el fin de esbozar un cuadro histórico, político, sanitario y cultural detallado del fenómeno. El análisis histórico y socio-psicológico del trabajo recorre las etapas del estigma del "loco" (desde la antigüedad, de hecho, la locura y la diversidad han sido dos de los problemas más desconocidos y frecuentes en la historia de la humanidad) desde la Edad Media hasta la actualidad, un periodo muy largo en el que ha habido cada vez más interpretaciones sobre el tema; baste decir que ahora se habla de "enfermedad mental", mientras que hace años el enfermo mental era denominado por la mayoría como "loco", víctima de la locura, una enfermedad incurable. A continuación, el análisis se realiza desde un punto de vista social y político, centrándose en los aspectos más oscuros y ocultos de los asilos "goffmanianos" definidos como "instituciones totales". En este sentido, la obra se centrará en las distintas polémicas y protestas a lo largo de los años, y en las posteriores leyes adoptadas al respecto, hasta llegar a la Ley Basaglia de 1978. También examinará en profundidad la organización interna de los manicomios, su estructura, exigua y desprovista de elementos perturbadores para los pacientes, las prácticas de formación del personal, sin dejar de lado, obviamente, las condiciones, la rutina diaria de los internos y, sobre todo, los "tratamientos especiales" reservados a los reclusos. La joya de la corona de esta obra es una entrevista con una antigua enfermera del mayor asilo de Italia, el de Mombello (MB), cuyo objetivo es recoger un testimonio tan directo como envolvente y, sobre todo, no exento de referencias empíricas. 


\section{Intervista ad un'ex infermiera del manicomio di Mombello}

Per i più, quando si parla di manicomi, sembra di parlare di qualcosa di molto remoto, ormai passato, eppure soltanto qualche decennio fa tali strutture erano ancora in attività in tutta la penisola italiana.

Chi non ha mai avuto a che fare con un manicomio può soltanto immaginare ciò che ivi accadeva, anche e soprattutto a causa di opere cinematografiche o romanzi: camicie di forza, catene, finestre con grandi sbarre, le classiche vestaglie bianche e chi più ne ha più ne metta.

Ma in realtà, cosa accadeva davvero ai pazienti lasciati ai margini della società ed isolati in queste strutture di cura e/o di internamento, il più delle volte forzato?

Questo paragrafo dell'elaborato sarà interamente incentrato su un'intervista ad un'ex infermiera della sezione femminile del manicomio di Mombello, con la quale ho avuto l'opportunità di dialogare in prima persona su sensazioni ed episodi realmente accaduti durante lo svolgimento della propria professione. Per rispetto della privacy non sarà rivelata l'identità dell'intervistata.

\section{Per quanto tempo ha lavorato nel manicomio di Mombello?}

"Non ricordo precisamente l'anno in cui presi servizio nel manicomio di Mombello, ma so che ci ho lavorato per più di 30 anni, se non vado errata credo di averci lavorato per 34 anni."

\section{Le piaceva il suo lavoro?}

"Si, mi piaceva molto lavorare come infermiera in quella struttura. La mia missione era di prestare soccorso a chi ne aveva bisogno. Anche le mie colleghe mi hanno fatto apprezzare $i$ lati positivi del mio lavoro."

\section{Cosa le piaceva del suo lavoro?}

"Tutto, mi piaceva tutto."

Com'era il manicomio nel momento in cui lei ha preso servizio per la prima volta?

"Scioccante."

\section{In che senso scioccante?}

"Nel senso che vedevo tutta questa gente con evidenti problemi in un ambiente non comune, ci sono rimasta male. Non male, ma come dirti, sono rimasta davvero scioccata. Vedevo gente che se la faceva addosso, gente sdraiata a terra, gente che gridava. Non tutti coloro che come me sono entrati in queste strutture hanno proseguito la loro professione, perché l'impatto con questo mondo diverso, ripeto, è scioccante."

\section{Cos'è che la scioccava tanto?}

"Mi scioccavano gli ammalati prima di tutto, perché dovevano essere contenuti, non tutti potevano girare liberamente, molti si accasciavano a terra, litigavano tra loro. Il primo giorno è stato quello più scioccante per me, così come credo che lo sia stato per tutti. Già dal secondo giorno in poi ho iniziato ad immergermi in questo nuovo mondo."

\section{C'era solidarietà tra infermieri sul lavoro? Vi aiutavate tra di voi?}

"Non tutte eravamo complici tra di noi, ma nella mia squadra abbiamo fin dal principio provato ad aiutarci. Altre squadre invece litigavano tra loro, ma ripeto, nella mia squadra c'era molta solidarietà, ci aiutavamo."

\section{Con quanti pazienti avete avuto a che fare?}

"Tantissimi, non ricordo precisamente il numero, ma nel mio padiglione c'erano almeno 400 pazienti. Erano troppi, ricordo che a volte molti dormivano addirittura nei corridoi, sempre sotto stretta sorveglianza, non potevano essere mai lasciati da soli."

\section{Potrebbe raccontarci la giornata tipo di un infermiere?}

"In realtà non c'era una giornata tipica. Tutto dipendeva dal compito che ti veniva assegnato. Ad esempio, quando venivo assegnata in infermeria, avevo a che fare con le allettate, la prima cosa che dovevo fare era lavare e cambiare le pazienti. Successivamente c'era il momento della colazione, bisognava imboccare le pazienti e far di tutto per loro, evitare che si sporcassero, e nel caso dovevo pulirle o cambiarle nuovamente.

Dovevamo rifare i letti, e se questi erano sporchi dovevamo cambiare le lenzuola. Ricordo che a rotazione, le pazienti avevano diritto a 2 o 3 bagni, e toccava a noi infermiere lavarle e rivestirle. Finito ciò, dovevamo somministrare le terapie prescritte dai medici ad ogni paziente.

Poi si pranzava, dovevamo tenere in posizione eretta le pazienti allettate che non riuscivano a sostenersi in maniera autonoma, dovevamo mettere i bavagli, le imboccavamo e le sistemavamo. Dopo pranzo dovevamo nuovamente somministrare la terapia.

Nel pomeriggio si faceva la merenda, dovevamo frullare la frutta per le nostre pazienti e fargliela mangiare. Soltanto le allettate avevano diritto alla merenda, le altre no. Queste ricevevano la merenda soltanto quando qualcuno dei loro parenti veniva a portarci un tegamino con su scritto il nome della propria cara. In questo caso anche la fortunata poteva fare merenda.

Nel corso di tutta la giornata dovevamo far fare i bagni alle pazienti. Arrivava la cena e punto e accapo.

Quando facevo il turno di notte dovevo mettere a letto le pazienti, e se qualcuna di loro aveva fatto il bagno dovevo prima asciugarla. Quando erano tutte a letto noi infermiere dovevamo controllare che tutto fosse tranquillo, ma non era mai così. C'era sempre qualcuna che aveva bisogno di assistenza, qualcuna che urlava o che si agitava. Dovevamo calmarle e farle bere acqua. Facevamo sempre il giro di tutti i letti insieme alla suora, se una paziente non era a letto bisognava andare a cercarla, anche se era andata in bagno, perché dovevano essere al proprio posto, come $i$ carcerati. Alle 4 del mattino dovevamo iniziare a cambiarle di nuovo, fino alle 7 del mattino, quando ci davano il cambio turno.

Quando invece non avevo servizio in infermeria il mio compito era di rendere presentabili le pazienti, dovevamo tagliarle $i$ capelli, tagliare le unghie di mani e piedi, dovevamo fare davvero di tutto.

Un altro giorno ci toccava lavare $i$ piatti, un altro giorno dovevamo pensare al bucato, ogni giorno era diverso, non avevamo sempre lo stesso lavoro.

Ricordo che non potevamo lavare i capelli a tutte tutti i giorni, c'erano dei turni. Usavamo la pettinina per pettinarle, il problema era quando c'erano i pidocchi e dovevamo spidocchiarle.

Le pazienti potevano entrare nel soggiorno comune soltanto quando si pranzava e quando si cenava, quelle più tranquille potevano passeggiare per $i$ corridoi o stare sedute in giardino. Queste più tranquille ci aiutavano anche a sparecchiare, portavano i piatti e le posate nel lavandino. Dovevamo lavare tutti i piatti a mano perché prima non c'erano le lavastoviglie.

Ricordo che c'erano pazienti che dovevano andare in chiesa, dovevamo accompagnarle. Le facevamo uscire una per volta e le 
contavamo, arrivati in chiesa dovevamo contarle nuovamente, perché se qualcuna scappava dovevi andare a cercarla. Finita la messa le accompagnavamo di nuovo nel dormitorio e dovevamo contarle, se mancava qualcuno ovviamente dovevamo andare sempre a cercarla.

Quando le pazienti avevano bisogno di un intervento dovevamo accompagnarle dal chirurgo.

Una volta non c'erano le siringhe usa e getta, bisognava sempre lavarle, sterilizzarle e asciugarle. Queste siringhe erano di varie dimensioni."

\section{Ci potrebbe raccontare un episodio particolare di qualche paziente che le è rimasto impresso?}

"Si, di una soprattutto. Lei era una ragazza molto giovane, ma soprattutto molto agitata. Era minuta, ma ci sono volute sette di noi per fermarla, era il diavolo in persona. L'abbiamo presa e legata a letto perché era fuori di senno, ma lei nonostante fosse legata mani e piedi riusciva comunque a spostare il letto.

Tutte le altre sono uscite dalla stanza a prendere qualcosa, io invece rimasi lì con lei. La ragazza mi chiamò per nome e mi chiese: "dimmi, io che male ho fatto per stare qui così?". Ci sono rimasta male, e mi è venuto da chiedermi cosa effettivamente avesse fatto di male questa ragazza per trovarsi li in queste condizioni. La poverina era schizofrenica, aveva sì dei momenti di lucidità, ma era spesso imprevedibile.

Ricordo che un giorno ero li chi mi stavo lavando le mani insieme ad una mia collega e la ragazza è entrata di nascosto. Né io, né la mia collega l'abbiamo sentita né vista arrivare. Ad un tratto mi è arrivato uno schiaffo cosi forte che io sono rimasta inerme. La mia collega ha iniziato ad urlare e per fortuna sono arrivate altre due colleghe. Abbiamo dovuto immobilizzarla, era una persona imprevedibile, non soltanto con me, ma proprio con tutti.

È stata l'unica paziente che ha "battezzato" sia noi infermiere che medici e professori che lavoravano con noi. Nessun'altra si era mai permessa di picchiare un medico o un professore perché ne avevano timore, ma lei sì, per lei eravamo tutti uguali, non aveva paura di nessuno. Non dava solo ceffoni, ma anche calci e pugni, e quando provavamo a fermarla si dimenava come se fosse il diavolo in persona. Anche quando arrivavano gli uomini facevano fatica a tenerla, e ripeto, lei era minuta.

Un'altra volta, sempre lei è andata di corpo nel letto. Io e un'altra mia collega siamo andate li per pulirla. Lei era sotto le coperte, e appena tirato su il lenzuolo la ragazza aveva le sue feci in mano e nel mentre diceva: "queste sono per mio figlio". Non vi dico le lotte che abbiam fatto per farla alzare, lavarla e pulire il suo letto.

Questa ragazza mi faceva tanta pena, parlava sempre di bambini. Si vociferava che suo padre fosse un alcolista, e che una sera, in preda ai fiumi dell'alcool l'avesse violentata e messa incinta. Ma questo è ciò che si vociferava, non era scritto nella sua cartella quando è arrivata nella nostra struttura. Forse però non tutto veniva scritto nelle cartelle delle pazienti.

Ricordo che sua zia le voleva un bene da matti, veniva a trovarla proprio tutte le domeniche. La ragazza si lasciava molto andare con sua zia, era calma e tranquilla con lei.

Un'altra storia che non dimenticherò mai riguarda un'altra ragazza che non era una "sciagurata", nel senso che non si lasciava andare o incline all'ignoranza. Era una ragazza abbastanza fine e tranquilla. Le piaceva leggere tanti libri nella nostra biblioteca presente nella struttura.

Un giorno, mentre io e le mie colleghe stavamo rifacendo i letti al piano di su, non ci siamo accorte che nella nostra stanza era entrata questa ragazza. Lei si era nascosta e si è buttata giù dalla finestra ed è morta. Ci siamo accorte di lei soltanto perché mentre si è buttata giù si è messa a gridare.
Non sappiamo come la ragazza abbia fatto a salire su, perché era sempre tutto chiuso, sia la porta al piano inferiore sia quella del piano superiore. Anche per aprire le finestre bisognava salire su uno sgabello, perché le finestre erano alte, e soprattutto non c'erano maniglie, noi infermiere avevamo un passe-partout, forse potrebbe esserci stata una dimenticanza tra qualcuna di noi."

\section{Ha mai avuto timore di qualche paziente?}

"Ce n'erano tanti che potevano metterti paura, dovevi stare attenta anche a come ti giravi, dovevi tener d'occhio tutta la situazione. C'erano anche quelli che non facevano niente, di loro non c'era da preoccuparsi, ma molti erano quelli potenzialmente pericolosi. Ho iniziato ad avere un po' più di timore quando da noi sono arrivati pazienti uomini, temevo che potessero alzarmi le mani e farmi del male."

\section{Ha mai percepito come un'ingiustizia la permanenza di una paziente nel vostro manicomio?}

“Ricordo che c'era un bel po'di via vai, molto spesso arrivavano da noi persone che non avevano malattie mentali, ma semplicemente avevano il vizio di bere o praticavano prostituzione. Questi arrivavano, e dopo qualche giorno venivano dimessi, ma per alcuni di loro tutto ciò era diventata un'abitudine, spesso tornavano da noi dopo due o tre giorni.

Ricordo in particolare di una paziente che è stata portata da noi e nessuno più è venuta a trovarla o a riprenderla. A quanto pare aveva una figlia, anche lei epilettica e ricoverata a Bergamo. Questa donna forse era più in forma di me, non assumeva farmaci, era pulitissima, ci aiutava a lavare la biancheria, si dava da fare per aiutarci. Noi le offrivamo sempre qualcosa, ad esempio quando andavano a fare il caffè gliene porgevamo sempre una tazza. Non l'ho mai vista fuori di sé, non l'ho mai vista sporca. Era sempre pulita e a modo.

C'era anche un'altra paziente che secondo me non doveva restare nella nostra struttura. Lei beveva, era un'alcolista, anch'essa, come l'altra donna, aveva una figlia epilettica ricoverata nella nostra struttura. L'indomani, smaltita la sbornia, la donna fu dimessa, mentre la figlia rimase li. Ricordo che questa donna pur di star vicino alla figlia è andata ad un bar lì vicino, si è ubriacata di nuovo. Sono andati a prenderla e così lei è rimasta ancora li con sua figlia, pur di non perderla. Lei si prendeva molta cura di sua figlia, la pettinava, la teneva sottobraccio.

Altre due donne, sempre madre e figlia, erano matte tutte e due. La mamma era una prostituta. La figlia invece aveva preso la sifilide. Erano state portate nella nostra struttura, ma le due non avevano un buon rapporto ed erano sempre messe in stanze diverse. Appena si incrociavano, anche per sbaglio, iniziavano a litigare e si "attaccavano" tra di loro. Loro non erano pazze, ma spesso ci capitava di accogliere nella nostra struttura anche prostitute e alcolisti."

\section{Come venivano definiti i vostri ospiti? Li chiamavate per nome?}

"In generale noi li chiamavamo pazienti o ammalati. Singolarmente invece li chiamavamo con il loro cognome."

\section{Al di là della legge Basaglia, si era già resa conto che alcuni metodi, come l'elettroshock, potessero essere deleteri per i pazienti?}

"In realtà no, non credo. Non so se da noi dopo la legge Basaglia veniva praticato ancora l'elettroshock, forse lo si faceva in altri ospedali, forse lo facevano anche dopo la legge Basaglia. Io personalmente non l'ho mai visto fare, ho portato i malati in sale d'osservazione dove c'era la macchina per fare l'elettroshock 
ma non vi ho mai assistito in prima persona. Però, come dire, secondo me era sì un male per i pazienti, però forse era un bene perché scuoteva i pazienti, soprattutto coloro che magari non volevano mangiare o non volevano fare nulla. Ad esempio, le pazienti depresse che rifiutavano ogni tipo di terapia e cibo erano coloro che venivano sottoposte ad elettroshock. Dopo questa terapia noi infermiere vedevamo la paziente molto più sveglia, iniziava a mangiare, seguiva la terapia. In questo tipo di persone depresse il risultato si vedeva, non era chissà che cosa, ma il risultato c'era, perché ripeto, prima dell'elettroshock non voleva toccare cibo o seguire la terapia, dopo invece si."

\section{È rimasta in contatto con qualche paziente dopo la chiusura della struttura?}

"No, non sono rimasta in contatto con nessuna delle mie pazienti, non ho più visto nessuna di loro."

\section{Quanto la sua esperienza professionale ha influito sulla sua vita privata?}

"Per niente, sono sempre riuscita a tenere fuori il mio lavoro dalla mia vita privata, ho sempre separato le due cose. Uscivo da Mombello e sapevo di tornare di casa mettevo da parte tutto ciò che mi capitava durante il giorno. Mentre quando uscivo di casa per andare a Mombello il lavoro era la prima cosa, e pensavo solo a quello."

\section{Qualcuno è mai guarito totalmente dalla propria malattia mentale?}

"Negli anni in cui ho lavorato io non ho mai visto nessuno guarire dalla propria malattia. Quelli più tranquilli a volte entravano e uscivano, ma in ogni caso rientravano sempre in struttura, nessuno è mai uscito definitivamente, almeno per quello che ne so."

\section{Tornando indietro nel tempo, sceglierebbe nuovamente di lavorare nel manicomio di Mombello?}

"Si, assolutamente, anche se era pesante, e ogni tanto ho preso le botte, tornerei sicuramente a lavorare li. Mi sentivo in dovere di aiutare le persone che, non per colpa loro, erano più sfortunate di me."

\section{Trattamento degli internati}

Ci troviamo sicuramente dinnanzi all'aspetto più avvolto di mistero, tetro e cupo di tutta la storia manicomiale. Tutto ciò che ci è dato sapere è stato portato alla luce dal personale che ivi lavorava e dagli archivi storici degli ormai ex manicomi, fonte preziosa di informazioni.

Da quest'ultime è emerso che le terapie praticate sui pazienti sono state fin troppo spesso "sperimentali", come se queste strutture fungessero da laboratori e i pazienti da cavie per esperimenti ai limiti dell'umanità (lobotomia, doccia fredda, elettroshock, apparecchi immobilizzatori come camice di forza).

In origine $i$ trattamenti sembravano essere i più disparati, quasi prescritti a caso da chi di competenza, tant'è che Elaine Murphy, membro della Camera dei Lord britannica, denominerà questa procedura "metodo culinario": "un pizzico di questo, una goccia di quello, una piccola dose di tartrato di antimonio, canfora, giusquiamo, morfina, lozioni rinfrescanti per la testa, vescicanti sulla nuca, ma con moderazione un po' di canapa indiana". ${ }^{1}$

Ovviamente tutto ciò non poteva avere alcun effetto, gli esiti si rivelavano sempre dubbi e inefficaci, pertanto ad esse si affianca- vano sempre altri trattamenti ritenuti validi per riportare alla normalità le condizioni dei propri pazienti. Tali soluzioni prevedevano delle misure di contenimento estreme, come ad esempio l'isolamento per un periodo non specificato e l'idroterapia, ma anche una pratica abbastanza innovativa per l'epoca, l'ergoterapia.

Per quanto riguarda l'isolamento (collocazione di un paziente da solo, contro la sua volontà, in una stanza chiusa a chiave), Esquirol, psichiatria e scienziato francese, sosteneva che tale pratica avesse dei benefici terapeutici sulla mente disturbata del paziente, che potevano essere risolti tramite la calma e il silenzio.

In questo caso i pazienti venivano rinchiusi in celle di isolamento o confinati in quartieri di isolamento, spesso con addosso una camicia di forza, strumento che costringe le braccia ad essere incrociate sul petto vietandone qualsiasi movimento e dalla quale è impossibile liberarsi a causa di forti cinghie sita sulla schiena. Essa veniva usata per placare gli attacchi nervosi e aggressivi dei pazienti, in modo che non potessero più muoversi. Tale strumento di contenimento fu usato per la prima volta in Francia nel 1770, per calmare pazienti schizofrenici o con frequenti disturbi d'ansia.

"Si riteneva che con la calma e il silenzio la mente tormentata potesse purificarsi e trasformarsi in una tabula rasa psicologica, pronta ad accogliere $i$ nuovi pensieri assennati che l'alienista vi avrebbe impiantato." 2

Oltre alla camicia di forza, spesso all'isolamento erano sovrapposte altre cure e rimedi, come ad esempio la somministrazione da parte dei medici al paziente di bromuro o di valeriana, usati entrambi come dei calmanti. Nel caso in cui l'effetto non fosse stato quello desiderato, la soluzione estrema sarebbe stata il semplice aumento delle dosi.

Soltanto a metà Ottocento ci si rese conto dell'inutilità di tali terapie nel contrasto alla malattia mentale. Pertanto, i neurologi e gli alienisti più "coraggiosi" cominciarono a sperimentare nuovi metodi di cura che potessero avere un più efficacia. A tal proposito iniziò l'utilizzo massiccio di sedie rotanti, docce alla testa, shock improvvisi e bagni freddi, ma anche quest'ultimi, che rimasero in voga per un gran bel lasso di tempo, non diedero mai dei veri e propri risultati.

Per calmare i malati era molto pratica la tecnica dell'idroterapia, che consisteva nell'immergere l'alienato in una vasca di acqua con una temperatura compresa o tra gli 8 e i 20 gradi centigradi quando questi era agitato.

Questa terapia poteva durare poche ore o addirittura, nei casi più estremi, anche un giorno intero. L'idroterapia era molto in voga nei primi anni del ventesimo secolo, ma la cosa sconcertante è che questa pratica non veniva intesa come una punizione, bensì era credenza comune tra neurologi e alienisti che l'acqua fredda avesse delle proprietà curative e benefiche per il paziente; si stimava che tramite questa procedura fosse possibile diminuire le attività cerebro-fisiche dell'alienato.

Al contrario, in alcuni casi il paziente veniva immerso in una vasca con dentro acqua calda che oscillava tra i 33 e i 36 gradi centigradi. Anche in questo caso, il paziente veniva lasciato in ammollo per ore o per tutta la notte. ${ }^{3}$

Murphy E., L'Ottocento: scienze mediche. Psichiatria e istituzioni, in «Storia della scienza, Treccani.it», 2003. https://www.treccani.it/enciclopedia/1-ottocento-scienze-mediche-psichiatria-e-istituzioni_\%28 Storia-della-Scienza\%29/

2 Murphy E., L'Ottocento: scienze mediche. Psichiatria e istituzioni, in «Storia della scienza, Treccani.it», 2003. https://www.treccani.it/enciclopedia/1-ottocento-scienze-mediche-psichiatria-e-istituzioni_\%28 Storia-della-Scienza\%29/

3 Ibidem. 
Come suddetto, in alcuni manicomi veniva praticata anche l'innovativa pratica dell'ergoterapia ovvero un "metodo curativo, complementare di altri trattamenti somatici o psicoterapici, in cui l'agente terapeutico è costituito da un'attività lavorativa razionalmente ordinata"4. Pionieri di tale pratica furono il già citato Philippe Pinel e Vincenzo Chiarugi (medico italiano vissuto a cavallo tra il diciottesimo e diciannovesimo secolo), che sperimentò l'ergoterapia nel manicomio di S. Bonifacio a Firenze con ottimi risultati, a differenza delle pratiche inumane utilizzate in precedenza.

Il fine di questa terapia era quello di far distrarre i malati dai loro pensieri ossessivi, facendoli sentire utili per la società attraverso il dovere, il lavoro e la produttività. Di riflesso essa risultava anche utile per il manicomio stesso, in quanto tramite il lavoro degli internati si poteva procedere all'autosostentamento.

Purtroppo, non tutti i manicomi erano provvisti di sale di lavoro, pertanto questa tecnica non fu applicata ovunque. Le istituzioni che al contrario possedevano delle strutture atte al lavoro proponevano agli internati numerose attività. Gli internati più tranquilli potevano essere d'ausilio agli infermieri nei compiti più elementari, mentre, nel caso in cui vi fosse stata la possibilità, i pazienti avrebbero potuto svolgere il lavoro che facevano nella realtà esterna prima di essere internati.

Nel corso del tempo la psichiatria si è evoluta, e con essa si sono sviluppate nuove terapie e trattamenti per i malati mentali, come la lobotomia, praticata per la prima volta a ridosso del 1900 , e l'elettroshock, scoperto e sviluppato da due neurologi italiani negli anni 30 del Novecento.

\section{Terapie estreme: elettroshock e lobotomia}

L'elettroshock, denominato anche "terapia elettroconvulsivante", è un trattamento terapeutico utilizzato in psichiatria, consistente nell'induzione di una convulsione nel paziente mediante una il passaggio di corrente elettrica attraverso il cervello. ${ }^{5}$

Questa nuova forma di terapia fa la sua comparsa nel panorama mondiale nel 1938, allorquando, dopo aver provato tale strumento su animali, Ugo Carletti e Lucio Bini (neurologi italiani), sperimentarono questa terapia su un paziente delirante, soggetto a depressione e in preda ad allucinazioni. Quest'ultimo, in seguito ad una serie di elettroshock riuscì a ritornare alla sua vita normale e riprese il lavoro. ${ }^{6}$

Tuttavia, non è tutto oro quel che luccica, infatti a fronte dei molti casi in cui la terapia funzionava efficacemente, se usato in maniera persistente e obsoleta, questo strumento avrebbe potuto portare gravi conseguenze per il paziente.

Essendo al tempo una scoperta innovativa, l'elettroshock cadde nel paradigma di uso-abuso: esso veniva applicato non solo su pazienti schizofrenici o affetti da disturbi affettivi, ma su qualsiasi persona che avesse un qualsiasi tipo di disturbo e ancor di più su drogati e alcolisti.

Da tener ben presente che tale trattamento ebbe origine prima della messa in commercio degli anestetici e veniva pertanto applicato su pazienti coscienti.

A causa del forte dolore e delle scosse elettriche indotte nel cervello, i pazienti perdevano conoscenza e andavano spesso incontro ad irrimediabili effetti collaterali, come fratture ossee e danni persistenti per il cervello e per il corpo. ${ }^{7}$

Queste le parole di un'anonima donna che ha visto portarsi via il padre da bambina in seguito all'internamento in un ospedale psichiatrico e conseguente elettroshock:

"Fermo come una cosa sorda all'acqua, cieca al sole.
Immobile come lo sono quegli oggetti violati e gettati nel pattume. La chimica dirigeva i suoi pensieri, i suoi circuiti neuronali semplici e mozzati. Gli occhi roteanti erano il solo ricordo di un uomo che era stato vivo, prima che le scariche di corrente penetrassero nei pensieri più nascosti [...]". Io non sapevo cosa fosse un padre pazzo;

[....] Lo videro alzare la mano livida e passarsi le dita sul capo, come a pettinarsi. Inconsapevole del suo sguardo bruciato, certo di un viso che non era più tale. Tossendo si levò in piedi e strisciò sul muro cercando nelle pietre la forza per alzarsi. Non volle specchi, solo il vecchio maglione felpato. Aprirono la porta intimoriti e io, lesta, fuggi dalla loro morsa correndo incontro a mio papà chiamandolo per nome. Mi feci prendere e alzare, sentendo quell'amore paterno che le scariche non avevano bruciato", 8

Nonostante al giorno d'oggi non si parli più di elettroshock, bensì di terapia elettroconvulsivante (TEC), e nonostante essa si sia evoluta nel tempo (le scariche non possono essere superiori a 5 volt ed inoltre deve essere praticata un'anestesia totale sul paziente), i dibattiti circa la brutalità, la mancata fondatezza scientifica e l'assenza di un comprovato valore terapeutico di questa pratica non sono mai cessati e soprattutto "non è chiaro quali $e$ in che modo queste modificazioni (dei neurotrasmettitori e dei meccanismi recettoriali) siano correlate all'effetto terapeutico". 9

In aggiunta all'elettroshock, anche la lobotomia fu uno degli esperimenti più invasivi e brutali sottoposti ai degenti in manicomio.

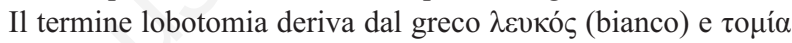
(taglio), e quindi "taglio della sostanza bianca, ossia delle zone cerebrali costituite da fasci di assoni mielinizzati, che appaiono bianche rispetto alle zone ricche di corpi cellulari e assoni non mielinizzati, che appaiono grigi." Essa consiste nel sezionare le connessioni nervose da e per la corteccia prefrontale, la parte più anteriore dei lobi frontali". ${ }^{10}$

La procedura utilizzata per praticare una lobotomia consisteva nel praticare un foro ad ambo i lati del cranio, dai quali in seguito doveva essere iniettato dell'alcool etilico puro che avrebbe dovuto agire nella porzione di materia bianca presente in prossimità della corteccia cerebrale.

Tale materia bianca è composta da parti di neuroni cerebrali, chiamate in gergo medico neuroni cerebrali, grazie alle quali gli impulsi elettrici vengono condotti dal nucleo del neurone interessato fino alle sue terminazioni.

Attraverso l'alcool era possibile disgregare tali collegamenti nervosi tra il cervello e il talamo.

www.treccani.it

www.treccani.it

Pancheri P., Caredda M., 1999, Elettroshock, in Universo del Corpo, Treccant.it. https://www.treccani.it/enciclopedia/elettroshock (Universo-del-Corpo)/\#: :text=\% $\%$ C3\%88\%20comunemente $\% 20$ denominata $\% 20$ elettroshock\%20(ECT,elettrica\%20veicolata $\% 20$ nelle $\% 20$ regioni $\% 20$ temporoparietali

7 Livi A., 27 Febbraio 2017, Elettroshock: la storia. https://www.alessandrolivistudiomedico.it/elettroshock-la-storia/

8 Montanari M., 12 Settembre 2019, Lo stigma intrappola i pazienti mentali. Ecco i racconti di chi è passato per l'elettroshock, pubblicato su "Il Fatto Quotidiano". https://www.ilfattoquotidiano.it/2019/ 09/12/lo-stigma-intrappola-i-pazienti-mentali-ecco-i-racconti-di-chie-passato-per-lelettroshock/5445686/

9 Cassano G.B., Manuale di psichiatria, Utet, Torino, 1994.

10 Dizionario di Medicina 2010, in Treccani.it. https://www.treccani.it/ enciclopedia/lobotomia_(Dizionario-di-Medicina)/ 
Si abusò fin troppo di questa "terapia" soprattutto negli Stati Uniti d'America, tant'è che negli anni '40 il numero dei pazienti che subì tale pratica fu ingente, senza una reale motivazione. ${ }^{11}$

Al giorno d'oggi la lobotomia è stata vietata dall'OMS (Organizzazione Mondiale della Sanità), per il semplice motivo che si possono ottenere risultati migliori con i farmaci, evitando tra l'altro anche i molteplici effetti collaterali. La lobotomia è praticata soltanto nel caso in cui dei pazienti non rispondano agli specifici e prescritti farmaci. ${ }^{12}$

\section{8: Legge Basaglia, la grande riforma e i suoi effetti sugli Ospedali Psichiatrici Giudiziari}

Prima di discutere sulla portata riformatrice della Legge 180/1978 mi sembra doveroso e opportuno presentare il pensiero di Franco Basaglia, soprannominato in seguito "dottore dei matti", colui che ha disposto la chiusura dei manicomi segnando una svolta nel mondo dell'assistenza ai pazienti psichiatrici, un vero e proprio distacco con il passato. A tal proposito citerò l'estratto di una discussione tenutasi durante un seminario che Basaglia tenne nel 1971 a Padova.

"A scuola abbiamo imparato a conoscere come psichiatri che i malati mentali, maniaci, schizofrenici o isterici, sono persone che difficilmente possono avere una evoluzione positiva e sono sempre in una situazione difettiva, per cui il loro destino è quello di restare in ospedale psichiatrico. Nell'istituzione troviamo delle persone che sono laceratori, sudici, lesionisti, spaccano tutto, sono pericolosi, ecc., $\grave{E}$ una popolazione che non ha niente a che fare con la popolazione che è fuori, è una popolazione che, si dico, sia tale perché è stata determinata dalla malattia; cioè abbiamo imparato che la persona che lacera, che si sporca, che è lurida, che è oscena, è tale perché la malattia produce questa situazione, questi sintomi. Ebbene, noi ci siamo chiesti se è possibile che delle persone perché sono malate debbano essere trattate in questo modo. Nell'ospedale psichiatrico il malato mentale è trattato come una cosa, come una bestia. Se il malato mentale è malato, noi ora ci spostiamo o andiamo a vedere un malato in un'altra istituzione medica, e vediamo che i malati che sono in questa istituzione sono e vivono come persone umane. Allora ci chiediamo se queste persone sono tali perché sono state ridotte dalla malattia in questo stato, oppure se sono tali perché l'istituzione le ha prodotte così. Questo è stato l'esperimento di un laboratorio vivente che noi abbiamo fatto a Gorizia nel '61. Abbiamo iniziato a fare quello che ritenevamo impossibile, cioè trasformare una istituzione da violenta e bruta e mortificante, in istituzione dove ci fosse la possibilità di chiamare un uomo "uomo".

Abbiamo cominciato questo lavoro di trasformazione e, a mano a mano che passavano gli anni, abbiamo visto come queste persone che ci erano state insegnate come irreversibili nella loro malattia, cambiavano faccia, e uno si chiama Antonio, uno Pietro, uno Nane, e non si chiamavano più malato di mente o numero 18; abbiamo cioè la possibilità di rendere una libera comunicazione in un mondo srealizzato, in un mondo strofizzato.

Cosa facciamo oggi di fronte a queste situazioni?

Io, oggi, dopo 10 anni sono nuovamente alle prese con le istituzioni psichiatriche. Sono direttore dell'ospedale psichiatrico di Parma, e vi trovo quella stessa realtà in cui mi sono trovato 10 anni fa nel tentativo di sovvertire quest'or- dine sociale interno per umanizzare e creare un nuovo tipo di organizzazione psichiatrica. Ho vinto il concorso a Parma e ho accettato Parma perché è in una "regione rossa" e speravo che l'Amministrazione Provinciale di questa città mi desse la possibilità di rompere più facilmente, più velocemente le strutture di questa istituzione. Peccato che questa regione sia in un contesto politico in cui l'Amministrazione Provinciale di Parma (o quella di Vicenza, per mettere due situazioni cosi agli antipodi) politicamente non dà la possibilità di trasformazione, perché fanno parte di uno stesso gioco che rende impossibile una reale trasformazione dell'istituzione.

E allora cosa dobbiamo fare? Lasciarci mortificare senza replicare?

Direi che quello che possiamo fare è continuare nella lotta perché pensiamo che la ragione sia dalla parte nostra. Diciamo che è assurdo che i malati mentali si trovino nella situazione in cui sono, cioè che dobbiamo trasformare queste istituzioni; però dobbiamo fare un'autocritica continua delle situazioni che viviamo.

La rivoluzione non si può fare, noi non la possiamo fare; ma il fatto è che dobbiamo trasformare la situazione, questa realtà nella quale noi viviamo, questa realtà invisibile che non è fatta certamente per noi, ma per altri di cui noi siamo i delegati, gli amministratori. Direi che c'è un passo in avanti da compiere: cioè questa visione un po' pessimistica ma reale della situazione ci induce a considerare cosa avviene quando la situazione socioculturale permette questa trasformazione istituzionale." 13

Nel 1971, Franco Basaglia assunse la direzione dell'ospedale psichiatrico della provincia di Trieste. Qui Basaglia istituì una prima comunità terapeutica, con l'obbiettivo di smantellare l'istituzione psichiatrica, vista dallo stesso Basaglia come "strumento di controllo". Ciò portò alla chiusura del manicomio di San Giovanni di Trieste nel 1977.

Furono questi anni di grande fermento per quanto riguarda la psichiatria, tant'è che nel 1978 la Corte Costituzionale si dichiara favorevole per il Referendum abrogativo degli articoli 1,2,3 e 3 bis della Legge 14 febbraio 1904, n. 36. Disposizioni sui manicomi e sugli alienati. Custodia e cura degli alienati, che fino ad allora disciplinava l'ambito manicomiale. Fu proprio tale scelta che portò ad una riforma totale sul trattamento dei malati mentali. Infatti, il 19 aprile il Consiglio dei ministri presentò il disegno di legge "Accertamenti e trattamenti sanitari volontari e obbligatori" che fu accolto ed approvato in sede legislativa il 2 e il 10 maggio, dopo sole tre settimane. Pertanto, il 13 maggio 1978 venne emanata la Legge 13 maggio 1978, n. 180, in tema di "Accertamenti e trattamenti sanitari volontari e obbligatori". L'iter legislativo fu fin troppo celere, infatti tale legge fu approvata a larghissima maggioranza dai parlamentari con la sola eccezione del gruppo dei Radicali, che si dichiararono contrari.

Si stima che l'iter sia stato così repentino anche perché l'Italia si trovava nel periodo dei cosiddetti "anni di piombo" ed anche e soprattutto perché il 9 maggio dello stesso, soltanto pochi giorni prima dell'approvazione della legge 180, nel bagagliaio di una

11 Ballero K.S., 22 Agosto 2017, La storia della lobotomia, tra narrazione e realtà pubblicato su Scientificast. https://www.scientificast.it/la-storia-della-lobotomia-narrazione-realta/

12 Ibidem.

13 Basaglia F., Antipsichiatria, presentato al seminario Franco Basaglia “Antipsichiatria”, Padova, 1971. 
Renault rossa ferma in via Caetani a Roma fu ritrovato, dopo ben cinquantacinque giorni, il corpo senza vita dell'allora Presidente della Democrazia Cristiana Aldo Moro, ucciso dalle Brigate Rosse. Ma questa è un'altra storia.

Nonostante i buoni propositi, la legge 180/1978 fu definita un “compromesso politico", poiché non si voleva troppo intaccare la legge 36/1904 attraverso il suddetto Referendum abrogativo.

Come detto in precedenza, la legge 180/1978 passerà comunque alla storia come «legge Basaglia, nonostante quest'ultimo ne rifiutò la paternità poiché essa non racchiudeva tutte le tesi "antipsichiatriche" da egli professate.

Celebre è l'intervista di Franco Giliberto a Franco Basaglia, nella quale il "dottore dei matti" riconosce le carenze e il carattere transitivo di tale norma. Queste le sue parole:

"Questa legge nasce come un compromesso per superare lo scoglio del referendum, che avrebbe eliminato una legge deprecabile, ma avrebbe lasciato un vuoto normativo che comunque andava colmato. Un compromesso politico quindi è quello che è. Esso va visto nell"ambito della volontà del governo di far rientrare questa normativa nella progettata riforma sanitaria. E" uno stralcio, una norma transitoria. Questo alimenta le perplessità sulle contraddizioni che la nuova normativa contiene, e le speranze che essa possa condurre a posizioni più avanzate [...]. Non bisogna lasciarsi andare a facili euforie. È una legge transitoria, fatta per evitare i referendum e perciò non immune da compromessi politici. Non si deve credere di aver trovato la panacea a tutti i problemi della malattia mentale con il suo inserimento negli ospedali tradizionali." 14

Considerata dai critici una legge incompleta ed incompiuta, la legge 180 fu una legge quadro che, effettivamente, presentava il limite della mancata definizione dei servizi e presidi alternativi all'Ospedale Psichiatrico e delle conseguenti linee guida.

Tra le critiche più aspre, ci fu quella dell'aver lasciato che $\mathrm{i}$ pazienti psichiatrici venissero scaricati sulle famiglie. ${ }^{15}$

Nonostante ciò, la Legge Basaglia portò indiscutibilmente notevoli modifiche a vantaggio delle persone affette da malattia mentale.

Come suddetto, per effetto della Legge venne predisposta la chiusura definitiva dei manicomi, stabilendo il graduale superamento degli ospedali psichiatrici e aprendo così la via alla rivoluzione in campo della salute mentale, non più contenitiva ma integrativa rispetto la società e riabilitativa verso la persona malata, nel rispetto dell'art. 27 della Costituzione Italiana.

Ad esempio, all'art. 1 è disposto che:

"Gli accertamenti e i trattamenti sanitari sono volontari.

Nei casi di cui alla presente legge e in quelli espressamente previsti da leggi dello Stato possono essere disposti dall'autorità sanitaria accertamenti e trattamenti sanitari obbligatori nel rispetto della dignità della persona e dei diritti civili e politici garantiti dalla Costituzione, compreso per quanto possibile il diritto alla libera scelta del medico e del luogo di cura. Gli accertamenti e i trattamenti sanitari obbligatori a carico dello Stato e di enti o istituzioni pubbliche sono attuati dai presidi sanitari pubblici territoriali e, ove necessiti la degenza, nelle strutture ospedaliere pubbliche o convenzionate. Nel corso del trattamento sanitario obbligatorio chi vi è sottoposto ha diritto di comunicare con chi ritenga opportuno [...]. "16

In quest'articolo è stabilito che tutti i trattamenti sanitari sono volontari, a sostegno dell'art. 32 della Costituzione Italiana. È ben noto come anche ci sia interesse nei confronti del paziente, tant'è che chi è sottoposto a TSO ha diritto di comunicare con chi egli ritenga opportuno e non sarà mai più internato.
Ora il "trattamento non volontario" viene chiamato Trattamento Sanitario Obbligatorio (TSO), e viene applicato soltanto in condizioni urgenti e di competenza ospedaliera. Può pertanto avvenire in determinate situazioni:

- Alterazioni psichiche tali da richiedere urgenti interventi terapeutici;

- Rifiuto delle cure da parte dell'infermo;

- Assenza di fattori e circostanze che consentano di adottare celeri e adatti interventi sanitari extra ospedalieri.

Altra grande novità riguarda lo spostamento è riscontrabile nell'art. 6, il quale recita:

"Gli interventi di prevenzione, cura e riabilitazione relati-

vi alle malattie mentali sono attuati di norma dai servizi e presidi psichiatrici extra ospedalieri.",17

È ben evidente lo spostamento extra ospedaliero della centralità funzionale del servizio. Ciò genererà quindi l'antefatto normativo utile alla creazione dei Centri di Salute Mentale (CSM) nella conseguente legge 23 dicembre 1978, n. 833, Istituzione del Servizio Sanitario Nazionale, la quale ingloberà la legge Basaglia. Inoltre, trattamento sanitario obbligatorio e i vari accertamenti in materia sono a carico dello Stato o di enti e istituzioni pubbliche.

Tuttavia, ci furono dei problemi di fondo nell'applicazione della legge. La chiusura degli Ospedali Psichiatrici Giudiziari ebbe anche conseguenze negative sulla società, in quanto un ingente numero di soggetti dapprima ivi reclusi per aver commesso crimini minori come risse e molestie, iniziò successivamente a gravare sulla società.

Si parlò perciò del problema della "criminalizzazione del malato psichiatrico", ciò sta a significare l'atto di recludere negli Ospedali Psichiatrici Giudiziari i "piccoli criminali", ovvero coloro che avevano necessità di interventi socioassistenziali, ma che l'autorità di polizia preferiva gestire tramite un ricovero medico psichiatrico piuttosto che attraverso l'attivazione di azioni penali.

$\mathrm{Si}$ accentuarono pertanto le pregresse problematiche della gestione interna degli OPG: se dapprima quest'ultimi avevano raccolto univocamente l'eredità dei manicomi civili, in seguito si ritrovarono a dover gestire altre funzioni come quella carceraria, in contrasto perciò con qualsiasi funzionalità terapeutica.

"Del resto che la popolazione degli Ospedali Psichiatrici Giudiziari fosse aumentata a seguito della legge 180, è confermato dai dati statistici: se fino a un anno prima della riforma il numero di internati era in costante diminuzione, passando dai 1.542 del 1970 ai 1.116 del 1977, nell' anno di approvazione della legge il numero iniziò a salire, prima di poche unità (erano 1.149 gli internati nel 1978) e poi sempre di più nei primi anni Ottanta, quando la riforma cominciò a produrre effetti concreti sulla realtà, toccando il picco nel 1982 con 1.600 persone ${ }^{18 "}$.

Il numero dei soggetti presenti all'interno degli allora chiamati manicomi e ora Ospedali Psichiatrici, crebbe notevolmente in seguito all'attuazione della legge Basaglia del 1978. Si noti che il numero totale di soggetti di entrambi i sessi nel 1978 ammontava a 1149 unità, numero sempre crescente di anno in anno (nel 1982 si toccò il picco con addirittura 1600 individui ricoverati).

14 Intervista a Franco Basaglia di Franco Giliberto, testo completo su Archivio storico de La Stampa, edizione venerdì 12 maggio 1978.

15 Basaglia F., Dall'apertura del manicomio alla nuova legge sull'assistenza psichiatrica, Giulio Einaudi Editore, Torino, 1982.

16 Legge 13 maggio 1978, n. 180, Accertamenti e trattamenti sanitari volontari e obbligatori.

17 Legge 13 maggio 1978, n. 180, Accertamenti e trattamenti sanitari volontari e obbligatori.

18 Andreoli V., Un secolo di follia, Milano, Rizzoli, 1991 
Nonostante ciò, in pochi mesi la legge 180/1978 venne inglobata all'interno della più ampia legge istitutiva del Servizio Sanitario Nazionale, la Legge 23 dicembre 1978, n. 833. Istituzione del Servizio Sanitario Nazionale.

Come suddetto, essendo quest'ultima una legge più ampia portò una serie di innovazioni maggiori:

- Fu eliminato il concetto di pericolosità per sé e gli altri e venne introdotto il trattamento sanitario in psichiatria basato sul diritto della persona alla cura e alla salute;

- Rispetto dei diritti umani come il diritto di comunicare e diritto di voto;

- Disposizione di chiusura degli ospedali psichiatrici su tutto il territorio nazionale;

- Costruzione di strutture alternative al manicomio;

- Ora i servizi psichiatrici territoriali divenivano il fulcro dell'assistenza psichiatrica, con conseguente territorializzazione della malattia.

- Furono istituiti i cosidetti Servizi Psichiatrici di Diagnosi e Cura (Spdc) all'interno degli ospedali generali per il trattamento delle patologie più gravi;

- Trattamento sanitario volontario, per il quale erano previsti prevenzione, cura e riabilitazione;

- Interventi terapeutici urgenti in caso di rifiuto di cure e mancanza di idonee condizioni per il trattamento extra-ospedaliero: Trattamento sanitario obbligatorio (TSO).

- Fu introdotto il concetto di "correlazione funzionale" tra Servizi Psichiatrici di Diagnosi e Cura e strutture di ricovero e servizi territoriali, sulla scia del principio di continuità terapeutica. ${ }^{19}$

Tuttavia, nonostante tutte le forze e le risorse impiegate in materia, fu soltanto dopo il "Progetto Obiettivo" del 1994 e con la conseguente messa in atto di misure e protocolli di assistenza psichiatrica da attivare a livello nazionale, che si raggiunse il termine ultimo di eliminazione dei residui manicominali. ${ }^{20}$

\section{Conclusioni}

La legge 180/1978 ha restituito diritto e dignità ad un numero ingente di uomini e donne che fino a quel momento erano rinchiusi nei manicomi (ospedali psichiatrici dal 1975). La critica mossa a questa legge, tuttavia, è stata dura; ciò che le si recriminava era il fatto di aver sì chiuso i manicomi, ma allo stesso ciò era stato visto come un segnale d'abbandono sia per i malati che per le proprie famiglie.

Nella legge infatti non erano presenti né linee guida né risorse apposite per reagire alle conseguenze derivanti dalla chiusura degli ospedali psichiatrici, tant'è che nelle varie regioni italiane, i servizi e le strutture sostitutive sono stati realizzati con tempi e risultati diversi.

Questo "falla normativa", nel tempo, ha portato pertanto a diverse criticità alle quali si deve rispondere con una buona dose di lucidità e consapevolezza, per evitare di prendere la strada più breve, la quale potrebbe far ritornare il nostro sistema alla vecchia logica manicomiale.

Trentasei anni dopo la chiusura degli ospedali psichiatrici esistono gli Spdc, sigla che sta per Servizio Psichiatrico di Diagnosi e Cura, "strutture di ricovero ospedaliero in cui viene garantita l'assistenza $\mathrm{H} 24$ e a cui afferiscono pazienti con disturbi psichici in fase acuta. L'indicazione al ricovero viene posta dai Dirigenti Medici del Dipartimento, presso le sedi dei Centri di salute mentale o presso le sedi di Pronto Soccorso, sulla base delle caratteristiche cliniche e sociali dei pazienti. I reparti, dotati di posti letto, effettuano ricoveri obbligatori (T.S.O.) e volontari (T.S.V.), secondo protocolli di cura condivisi." 21

Nonostante il Servizio Psichiatrico di Diagnosi e Cura sia per l'appunto un servizio molto più innovativo e razionale rispetto alle strutture del passato, anche in questi centri non sembra che vi sia un totale rispetto per i propri pazienti. Secondo Piero Cipriano, noto medico psichiatra e psicoterapeuta italiano, la cura dei pazienti presenti nei Servizi Psichiatrici di Diagnosi e Cura spesso si risolve attraverso due azioni, con l'uso di psicofarmaci e sedativi, e con delle fasce robuste che tengono i pazienti stretti a letto, proprio come avveniva nei vecchi manicomi. A tal proposito:

"Il manicomio ricordava un campo di concentramento, il Spdc ricorda una fabbrica. [...]. Per il ricoverato mentale è raro fare qualche forma di terapia che non sia inghiottire farmaci, perché in molti Spdc l'organizzazione prevede un solo medico che, essendo appunto solo, lavora spesso per le urgenze. La sera c'è la cena, la televisione, le pasticche, poi si deve dormire, e se il paziente non dorme prende la terapia aggiuntiva, perché il sonno in Spdc è sacrosanto, per i pazienti ma ancora di più per i medici e gli infermieri [...]. Qui ci sono ancora le fasce per legare i pazienti al letto, le sbarre alle finestre e le porte chiuse: tutti presupposti per la perdita della fiducia, che segna la fine di ogni percorso riabilitativo e la perpetuazione del principio matto "uguale pericoloso" [...]. ",22

Stando alle parole di Cipriano, sembrerebbe che pian piano, ovviamente non ovunque, si stia tornando alla vecchia logica manicomiale. Spesso queste mancanze sembrano esser causate dalla mancanza di personale addetto e qualificato, sia nelle strutture private sia soprattutto in quelle pubbliche in quanto: "al sistema pubblico sanitario le fasce costano meno degli operatori e la legge non le ha mai vietate. Quelli che le usano dicono che in mancanza di personale non si può fare altrimenti. Ma chi lavora nel settore sa bene che spesso un malato viene legato per motivi futili, come un urlo di troppo". ${ }^{23}$

Al netto dei fatti, ci si sta ancora interrogando su come e cosa fare per evitare che nel XXI secolo, e dopo ben quarantadue anni dalla legge Basaglia, tutto ciò sia ancora possibile.

Si potrebbe arrivare ad una soluzione multifattoriale, che tenga conto di fattori biologici, sociali e psicologici. Bisogna che sia una fusione tra la prevenzione, la cura e la riabilitazione con l'arte del socializzare e del lavorare. ${ }^{24}$

Sulla base di ciò, nel 2005, il Servizio di Salute Mentale di Trento ha deciso di far partire il progetto UFE, sigla che sta per Utenti e Familiari Esperti, dove gli utenti sono persone che in passato hanno sofferto di malattie mentali, i quali, con i loro familiari, oggi aiutano le persone che soffrono degli stessi disturbi con l'obiettivo di aumentare la loro partecipazione attiva nelle pratiche quotidiane. In questo progetto c'è la voglia del "fare assieme", infatti gli ex malati mentali o coloro che hanno imparato a gestirla, lavorano fianco a fianco con i dottori, il che porta a risvolti positivi sia per i dottori stessi, perché tramite queste nozioni riescono a rap-

19 Legge 23 dicembre 1978, n. 833. Istituzione del Servizio Sanitario Nazionale.

20 Basaglia F., Dall'apertura del manicomio alla nuova legge sull'assistenza psichiatrica, Giulio Einaudi Editore, Torino, 1982.

21 www.as13.liguria.it

22 Cipriano P., La fabbrica della cura mentale. Diario di uno psichiatra riluttante, Elèuthera, Milano, 2013.

23 Ibidem.

24 Cozza M., 8 Aprile 2018, I primi 40 anni della 180. Dalla chiusura dei manicomi alla fine degli Ospedali psichiatrici giudiziari. È il momento di una seconda Conferenza Nazionale per la Salute Mentale, pubblicato su "Quotidiano Sanità", https://www.quotidianosanita.it/studi-eanalisi/articolo.php?articolo_id $=60503$ 
portarsi meglio con i nuovi pazienti; che per gli UFE, poiché questi intraprendono un percorso e un lavoro che migliora la propria qualità di vita, e soprattutto, potranno far del bene agli altri malati psichici con i quali si rapportano.

La guarigione non è legata soltanto ad una terapia efficace, ma anche alle circostanze. Se la medicina normale combatte le malattie del corpo agendo direttamente su di esso, per contrastare la malattia mentale è fondamentale capire e accettare la propria condizione, perché è attraverso la consapevolezza di sé che è possibile percorrere un sentiero seppur tortuoso, ma pur sempre di graduale ascesa verso la guarigione.

\section{Bibliografia}

AA.VV, Il Recovery in Psichiatria. Organizzazione dei servizi e tecniche operative, Erickson, Trento, 2012.

Ambrosini G., Ceccarelli P. E, L'esclusione manicomiale e la sua legge, in Quale giustizia, 1972.

Andreoli V., Un secolo di follia, Milano, Rizzoli, 1991.

Babini, P.V., Liberi tutti: Manicomi e psichiatri in Italia: una storia del Novecento, Bologna, il Mulino 2009.

Baccarani U., Infermieri e infermiere, Modena, Società Tipografica Modenese, 1909.

Basaglia F., Che cos'è la psichiatria? a cura di Franco Basaglia, Baldini \& Castoldi, Milano, 1977.

Basaglia F., Conferenze brasiliane, a cura di Franca Ongaro Basaglia e Maria Grazia Giannichedda, Raffaello Cortina editore, Milano, 2000.

Basaglia F., Dall'apertura del manicomio alla nuova legge sull'assistenza psichiatrica, Giulio Einaudi Editore, Torino, 1982.

Basaglia F. L'istituzione negata, Torino, Einaudi, 1968,

Basaglia F., La distruzione dell'Ospedale Psichiatrico come luogo di istituzionalizzazione. Mortificazione e libertà dello "spazio chiuso". Considerazioni sul sistema "open door", estratto dagli annali di neurologia e psichiatria e annali Ospedale Psichiatrico di Perugia, 1965.

Bozzo L., Il codice penale e la sua genesi con note di giurisprudenza ed altre utili per la sua pratica, Roma, 1890.

Briciola F., Il carcere riformato, Il Mulino, Bologna, 1977.

Brunori L., Raggi C., Le comunità terapeutiche, Il Mulino, Bologna, 2007.

Canosa R., Storia del manicomio in Italia dalleeUnità deeItalia ad oggi, Milano, Feltrinelli, 1979.

Cappelli I., Il manicomio giudiziario, in Quale giustizia, 1972.

Carabelli P.G., L'infermiere di ospedale psichiatrico, Milano, Edito a cura dell'amministrazione Provinciale, 1937.

Carozza P., Principi di riabilitazione psichiatrica. Per un sistema di servizi orientato alla guarigione, Franco Angeli, Milano, 2006.

Cassano G.B., Manuale di psichiatria, UTET, Torino, 1994.

Catananti C., Nascita ed evoluzione dell'ospedale: dall'ospitalità alla organizzazione scientifica in Rapporto Sanità 2000: l'Ospedale del futuro, Il Mulino, Bologna, 2000.

Ceroni L., Manuale per gli infermieri degli ospedali psichiatrici, Como, Cavalleri, 1942.

Cipriano P., La fabbrica della cura mentale. Diario di uno psichiatra riluttante, Elèuthera, Milano, 2013.

Clemmer D., La comunità carceraria, in Carcere e società liberale (Santoro, E.), Giappichelli, Torino, 1997.

Curcio R., Valentino N., Petrelli S., Nel bosco di Bistorco. Sensibili alle foglie, Roma, 1997.

D'agostino A., Le città dimenticate. Dalla città per la cura alla cura per la città in Rapporto sullo stato degli ex ospedali psichiatrici in Italia, a cura di D'Agostino A., Festival dell'Architettura Magazine, ricerche e progetti sull'architettura e la città, 2017.

D'alessandro R., Lo specchio rimosso. Individuo, società, follia, da Goffman a Basaglia, Franco Angeli, Milano, 2008.

Da Rin M., Il lavoro fra alienazione e liberazione, Marsilio, Venezia, 1991.

De Giacomo U., Manuale per gli infermieri di ospedale psichiatrico, Edizioni Mediterranee, Roma, 1959.V.P. BABINI, Liberi tutti. Manicomi e psichiatri in Italia: una storia del Novecento, Bologna, Il Mulino, 2009.

Dolcini E., Rieducazione del condannato e rischi di involuzioni neoretributive: ovvero della lungimiranza del costituente, in Rassegna penitenziaria e criminologica, 2006.

Donghi D. (a cura di), voce manicomi, in Manuale dell'architetto, volume II, sezione III, (sez. II del capitolo XV - Stabilimenti sanitari), Torino, Unione Tipografico- editrice torinese, 1927.

Dotto G., Corso teorico e pratico per gli infermieri dell'ospedale psichiatrico, Palermo, Sc. Tip. Saler. Orf. Guerra, 1929.

Famiglietti L., Verso il superamento della pena manicomiale, in Giurisprudenza Costituzionale, 2003.

Ferrario P., Nobili dimore. Le residenze storiche a Limbiate e Mombello, Limbiate, Studio Archivolto e Comune di Limbiate, 2001.

Foot J., La "Repubblica dei matti". Franco Basaglia e la psichiatria radicale in Italia, 1961-1978, Feltrinelli Editore, Milano, 2017.

Fornari U., Trattato di Psichiatria Forense, UTET, Torino, 2008.

Foucault M., Il manicomio illimitato, in Follia e Psichiatria, a cura di M. Bertani e A. Rovatti, Giuffrè, Milano, 1994.

Foucault M., Malattia mentale e psicologia, Cortina, Milano, 1997.

Foucalt M., Nascita della clinica, Torino, Einaudi 1969.

Foucalt M., Sorvegliare e Punire. Nascita della Prigione, Einaudi, Torino, 1976.

Foucault M., Storia della follia nell'età Classica, Rizzoli, Milano, 1997.

Galimberti U., Dizionario di Psicologia, Utet, Torino, 1999.

Garofalo R., Criminologia. Studio sul delitto e sulla teoria della repressione, Fratelli Bocca, Torino, 1891.

Goffman E., Asylums: le istituzioni totali: i meccanismi dell'esclusione e della violenza. Torino: Einaudi, 1968.

Goffman E., Stigma. L'identità negata, Ombre corte, Verona, 2012.

Intervista a Franco Basaglia di Franco Giliberto, testo completo su Archivio storico de La Stampa, edizione venerdì 12 maggio 1978, pag. 11.

Lenza C., Il manicomio italiano nell'europa dell'ottocento. Gli esordi del dibattito e la questione dei modelli in AA.VV., I complessi manicomiali tra Otto e Novecento.

Libertini R., Il manicomio non ha mai fine, in Quale giustizia, 1974.

Lombroso C., La cattiva organizzazione della polizia ed i sistemi carcerari, 1900, in Il momento attuale, Milano, 1903, 94.

Lombroso C., L'uomo delinquente in rapporto all'antropologia, alla giurisprudenza ed alla psichiatria, Roma, 1897.

Lombroso C., Sull'istituzione dei manicomi criminali in Italia, in Rendiconti del Regio Istituto Lombardo di scienze, lettere e arti, 1872 .

Lupo E., Il progetto di modifica della legge 180: una controriforma fondata sulla segregazione, In Questione Giustizia, 2003.

Manna A., Imputabilità e nuovi modelli di sanzione. Dalle "finzione giuridiche" alla "terapia sociale", Giappichelli, Torino, 1997.

Marinucci G., Dolcini E., Manuale di Diritto Penale, Giuffrè, Milano, 2006.

Merlini S., Libertà personale e tutela della salute mentale: profili costituzionali, in Democrazia e Diritto, 1970.

Miravalle M., "La riforma della sanità penitenziaria: il caso 
Ospedali Psichiatrici Giudiziari. Esigenze etiche e giuridiche dell'oltre", tesi di laurea in Sociologia del diritto, Torino, 2011.

Modena G., Appunti di tecnica manicomiale, in Giornale di psichiatria clinica e tecnica manicomiale. Bollettino del manicomio di Ferrara, Ferrara, S.A. Industrie Grafiche, 1908.

Padovani E., Pinel e il rinnovamento dell'assistenza degli alienati, in Giornale di psichiatria clinica e tecnica manicomiale. Bollettino del manicomio di Ferrara, Ferrara, S.A. Industrie Grafiche, 1927.

PANTOZZI G., Storia delle idee e delle leggi psichiatriche, Centro studi Erickson, Milano, 1994.

PELISSERO M., Il definitivo superamento degli ospedali psichiatrici giudiziari nel tempo della crisi, in Dir. pen. e proc., 2012.

Rabaglitti G., Manicomi, in Novissimo Digesto, vol. X, UTET, Torino, 1957.

Relazione tenuta il 23 ottobre 1897 all'assemblea degli psichiatri e dei neurologi della Germania centrale ad Halle, dal Dr. Ganser di Dresda, tratta da Archiv für Psychiatrie und Nervenkrankheiten.

Roscioni L., Il governo della follia. Ospedali, medici e pazzi in età moderna, Milano, Bruno Mondadori, 2003.

Saporito F., (1908) "Su gl'incorreggibili e il loro governo razionale: nota di psicologia criminale", Aversa,1908.

Scabia L., Guida dell'infermiere dei malati di mente nella casa e nel manicomio, Torino, UTET, 1901.

Williams F.P. III, Mcshane M.D., Devianza e criminalità, Il mulino, 2002.

\section{Normative/Leggi}

Codice penale del Granducato di Toscana, 1853.

Codice penale per gli Stati di Parma, Piacenza e Guastalla,1820.

Codice penale per gli Stati di S.M. Il Re di Sardegna, 1839.

Codice penale per gli Stati di S.M. Il Re di Sardegna, 1839.

Codice penale per Lo Regno delle Due Sicilie, 1819.

La Legge 26 luglio 1975, n. 354 "Norme sull'ordinamento penitenziario e sulla esecuzione delle misure privative e limitative della liberta.

Legge 14 febbraio 1904, n. 36, Disposizioni sui manicomi e sugli alienati. Custodia e cura degli alienati.

Legge 18 marzo 1968, n. 431, Provvidenze per l'assistenza psichiatrica.

Legge 23 dicembre 1978, n. 833. Istituzione del Servizio Sanitario Nazionale.

Regio decreto $1^{\circ}$ dicembre 1889, n. 6509, Approvazione del Codice Penale.

Relazione a S.E. il Ministro dell ${ }^{e e}$ Interno sulla ispezione dei manicomi del Regno, 1891.

\section{Sitografia}

11 Aprile2014, L'elettroshock, pubblicato su "La coscienza di Zeno", http://s205992.blogspot.com/2014/04/ero-andato-daquel-medico-perche-mera.html

Avico R., 17 Giugno 2019, Asylums di goffman: per una cura consapevole in psichiatria, pubblicato su "Psicologia Fenomenologica". https://www.psicologiafenomenologica.it/ articolo/asylum-di-goffman-per-una-cura-consapevole-in-psichiatria

Balestrazzi C., 2018, Dalla nascita dei manicomi alla loro chiusura, pubblicato su "Associazione Idee In Circolo". https://associazioneideeincircolo.wordpress.com/2018/04/16/dalla-nascita-deimanicomi-alla-loro-chiusura/

Ballero K. S., 22 Agosto 2017, La storia della lobotomia, tra narrazione e realtà, pubblicato su "Scientificast". https://www.scientificast.it/la-storia-della-lobotomia-narrazione-realta/

Borzacchiello A., I luoghi della follia, l'invenzione del manicomio criminale. https:/www.museocriminologico.it/images/PDF/ Carcere/manicomio_criminale.pdf

Cadeddu G., 4 Marzo 2014, La cruda realtà dei manicomi, pubblicato su "Fotografismo". http://fotografismo.altervista.org/lacruda-realta-dei-manicomi/

Capriglio M., 5 Settembre 2020, L'ospedale psichiatrico nelle parole di un ex infermiere, pubblicato su "The wise magazine" .https://www.thewisemagazine.it/2020/09/05/ospedale-psichiatrico-ex-infermiere/

Collettivo Antipsichiatrico Antonin Artaud di Pisa, 11 Gennaio 2017, Elettroshock: ma quale cura? pubblicato su "Canto delle Sirene". http://www.cantodellesirene.altervista.org/?q=node/401

Colombo V., 2 giugno 2017, Infermiera ai tempi del "manicomio, pubblicato su "Sguardi di confine". https:/www.sguardidiconfine.com/ospedale-psichiatrico-varese-la-testimonianza-uninfermiera-manicomio/

Cozza M., 8 Aprile 2018, I primi 40 anni della 180. Dalla chiusura dei manicomi alla fine degli Ospedali psichiatrici giudiziari. È il momento di una seconda Conferenza Nazionale per la Salute Mentale, pubblicato su "Quotidiano Sanità. https://www.quotidianosanita.it/studi-e-analisi/articolo.php?articolo_id=60503

De Felice A., 2017, Il manicomio di Mombello, tra i luoghi maledetti della Lombardia, pubblicato su "Milano Weekend". https://www. milanoweekend.it/articoli/mombello-manicomio-visita/

Dizionario di Medicina 2010, in Treccani.it. https://www. treccani.it/enciclopedia/lobotomia_(Dizionario-di-Medicina)/

Fondazione Benetton Studi Ricerche, Per un atlante degli ospedali psichiatrici pubblici in Italia. Censimento geografico, cronologico e tipologico al 31 dicembre 1996. http://www.fbsr.it/wp-content/uploads/1994/09/Per-un-atlante-degli-ospedali-psichiatricilaboratorio-1994-ricerca.pdf

Foto di Roberto Bettolini ne "Il manicomio di Mombello", 5 Luglio 2017, pubblicato su "Storie Dimenticate".

https://www.asl3.liguria.it

https://www.camera.it/

https://www.giustizia.it/

http://www.ilfogliopsichiatrico.it

Livi A., 27 Febbraio 2017, Elettroshock: la storia. https://www.alessandrolivistudiomedico.it/elettroshock-la-storia/

Marcomin F., 08 Settembre 2020, Mombello era il manicomio più grande d'Italia. Uno dei luoghi più spaventosi del mondo, pubblicato su "Milano Città Stato". https://www.milano cittastato.it/evergreen/forse-non-sapevi-che/mombello-era-ilmanicomio-piu-grande-ditalia-uno-dei-luoghi-piu-spaventosidel-mondo/

Marra G, 11 Aprile 2017, Erving Goffman e il ruolo delle istituzioni totali, pubblicato su "Sociologicamente.it". https://sociologicamente.it/erving-goffman-e-il-ruolo-delle-istituzioni-totali/

Mantovani F., Esclusione sociale e violenza istituzionale, pubblicato su "Diacronie". http://journals.openedition.org/diacronie/1695

Murphy E., L'Ottocento: scienze mediche. Psichiatria e istituzioni, in Storia della scienza, Treccani.it, 2003. https://www. treccani.it/enciclopedia/1-ottocento-scienze-mediche-psichiatria-e-istituzioni_\%28Storia-della-Scienza\%29/

Pancheri P., Caredda M., Elettroshock, in Universo del Corpo, Treccanti.it. 1999. https://www.treccani.it/enciclopedia/elettroshock_(Universo-del-Corpo) 
Pelissero M., 2012, Il doppio binario nel sistema penale italiano. http://www.antoniocasella.eu/archipsy/Pelissero_2012.pdf

Treppo P., 16 Gennaio 2017, Vita da infermiere in manicomio, tra elettroshock, prostitute e ubriachi, pubblicato su "Il Gazzettino". https://www.ilgazzettino.it/nordest/udine/infermiere_manicomio_udine_elettroshock_prostitute_ubriachi-2198602.h. html

Turco M., La sindrome di Ganser: aspetti psicologici e forensi. http://www.forensicsgroup.it/doc/la_sindrome_di_Ganser.pdf

Correspondence: Carmine Caputo.

E-mail: c.carmine189605@gmail.com

Key words: asylum; insanity; public utility.

Parole chiave: manicomio; malattia mentale; pubblica utilità.

Palabras clave: manicomio; insanidad; utilidad pública.

Received for publication: 11 June 2020.

Accepted for publication: 28 July 2020.

This article is distributed under the terms of the Creative Commons Attribution Noncommercial License (by-nc 4.0) which permits any noncommercial use, distribution, and reproduction in any medium, provided the original author(s) and source are credited.

${ }^{\circ}$ Copyright: the Author(s), 2020

Licensee PAGEPress, Italy

Rivista di Psicopatologia Forense, Medicina Legale, Criminologia 2020; $25: 550$

doi:10.4081/psyco.2020.550 